\section{Structural and functional correlates in color vision deficiency}

\begin{abstract}
Purpose The aim of this study is to assess the photoreceptor integrity, using spectral domain optical coherence tomography (SD-OCT), and to measure the retinal sensitivity of patients with congenital red-green color vision deficiency (CVD).

Methods In all, 14 eyes from 7 patients
\end{abstract} with congenital red-green CVD (diagnosed by Farnsworth Munsell 100 hue test), and 14 eyes from 7 control subjects were examined by SD-OCT and microperimetry. Radial scans $(7-\mathrm{mm})$ were taken of the macula. The center of the fovea was defined. The thickness of different retinal layers, at the foveal center, and at multiple defined points along all six radial scans, was measured. The median readings were compared between the two groups using Mann-Whitney $U$-test.

Results SD-OCT demonstrated normal total retinal thickness, normal thickness of the photoreceptor layer, normal thickness of the outer nuclear layer, normal vertical thickness of the outer segments (OSs), and normal vertical thickness of the inner segments. OS horizontal diameter was less in left eye in cases with CVD when compared with controls. The mean retinal and foveal sensitivity was similar between cases and controls. Conclusions In subjects with congenital red-green CVD, there are no discernible anatomical abnormalities seen on SD-OCT in various retinal layers, except for a narrower foveal pit. However, further studies with larger sample size are required. Eye (2011) 25, 909-917; doi:10.1038/eye.2011.87; published online 15 April 2011

Keywords: color vision deficiency; photoreceptors; spectral domain optical coherence tomography; microperimetry
A Gupta, G Laxmi, MG Nittala and R Raman

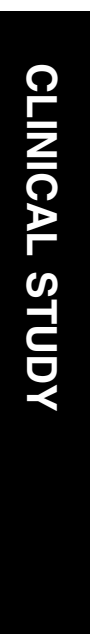

Thomas Young recognized at the beginning of the 19th century that normal human color vision is trichromatic. ${ }^{1}$ Normal color vision is derived from the presence of three spectrally distinct cone types: long, middle, and short wavelength sensitive ( $\mathrm{L}, \mathrm{M}$, and $\mathrm{S}$ ). The most common forms of color vision deficiency (CVD) are inherited and associated with the inability to discriminate between red and green wavelengths. Red-green CVD is characterized by the absence of either the L-cone function, known as protan defects (disturbances in the L-cone photopigment; with protanomaly being the alteration form and protanopia being the loss form) or the M-cone function known as deutan defects (disturbances in M-cone photopigment; with deuteranomaly being the alteration form and deuteranopia being the loss form). ${ }^{2,3}$ They can be linked to disruptions at the $\mathrm{X}$-chromosome opsin gene locus, where the L- and M-cone opsin genes reside in a head-to-tail array. ${ }^{4}$ Because these defects are inherited as $\mathrm{X}$-linked recessive traits, the incidences are much higher in Caucasian males $(8.0 \%)$ than in females $(0.4 \%))^{5}$ These molecular changes result in functional defects in color vision.

The Farnsworth Munsell 100 hue test (FM 100 test) is the most comprehensive of the FM type tests, giving both differential diagnosis and progression of the disease. ${ }^{6}$

Spectral domain optical coherence tomography (SD-OCT) is a newer generation high-resolution OCT with the advantages of high-speed data acquisition, three-dimensional reconstruction of acquired retinal images, and improved visualization of retinal architecture, layer by layer, including photoreceptor inner segment (IS)-outer segment (OS) ${ }^{7-10}$

Barthelmes et $a l^{11}$ studied the structural changes in the central retina on OCT in patients with two cone diseases-blue-cone monochromatism (BCM) and achromatopsia
Shri Bhagwan Mahavir Vitreoretinal Services, Sankara Nethralaya, Chennai, Tamil Nadu, India

Correspondence: A Gupta, Shri Bhagwan Mahavir Vitreoretinal Services, Sankara Nethralaya, Tamil Nadu 600 006, India Tel: +91442 827 1616; Fax: + 914428254180 . E-mail: guptaaditi_dr@ yahoo.com Accepted in revised form: 2 March 2011 Published online: 15 April 2011 18 College Road, Chennai,

Received: 29 June 2010 
$(\mathrm{ACH})$ and compared the results with the healthy control individuals. The study reported a highly altered foveolar structure in both BCM and $\mathrm{ACH}$. Varsányi et al ${ }^{12}$ reported reduced thickness of the central retina on OCT in patients with $\mathrm{ACH}$.

However, little is known about the structural changes in the retina in subjects with congenital CVD, which is also characterized by the lack of functioning cones. Carroll et $a l^{13}$ studied subjects having red-green CVD with C203R mutation and observed a partial thinning of the outer nuclear layer (ONL) in the retina of subjects with C203R mutation compared with a normal ONL in subjects with LIAVA mutation.

This study was conducted to examine in vivo the anatomic structure of the retina and to measure the retinal sensitivity of patients with congenital red-green CVD.

\section{Materials and methods}

In all, 14 eyes from seven patients with congenital CVD were examined at our department in 2009 by the SD-OCT and microperimetry (MP). The results were compared with the 14 eyes of seven healthy, age-matched control subjects.

The study was approved by the Institutional Review Board of the hospital and adhered to the tenets of the Helsinki declaration. An informed consent was obtained from all subjects to whom the nature and possible consequences of the study were explained. The participants underwent a standard ophthalmic examination. The best-corrected visual acuity (BCVA) was measured using standard Snellen eye charts. Snellen BCVA was converted to the log of the minimum angle of resolution (logMAR) for statistical analysis. Color vision was assessed by Ishihara and by FM 100 test. SD-OCT (Copernicus, Optopol Technologies, Zawiercie, Poland) was used to measure the thickness of the various retinal layers and MP (MP 1 Microperimeter, Nidek Technologies, Padova, Italy) was used to assess the retinal sensitivity.

\section{Spectral domain optical coherence tomography}

All the measurements were taken manually by a single experienced observer, and the manual assessment of the SD-OCT parameters was repeated twice by the same observer. The intra-observer repeatability was generally good ( $k=0.651$ for photoreceptor layer (PRL) and $k=0.976$ for FT). All OCT images were acquired through a dilated pupil. The gray scale images were used for precisely identifying and measuring the thickness of the various retinal layers. ${ }^{14}$ We used $7-\mathrm{mm}$ scan length with asterisk scan, protocol with 6 B-scans and 2743 A-scans per B-scan. The acquisition time was $0.76 \mathrm{~s}$. To evaluate and compare the thicknesses of the various retinal layers at the central fovea, between the normal controls and the patients, we chose the OCT image with the steepest foveal excavation from the asterisk scan across the foveal area. The foveal center was then defined in the chosen scan by a hyper-reflective dot echo at the innermost retinal layer.

Using the computer-based caliper measurement tool in the SD-OCT system, the thickness of the foveal neurosensory retina was measured by measuring the distance between the vitreo-retinal interface (hyperreflective echo of the innermost retinal layer representing internal limiting membrane (ILM)) and the inner edge of the retinal pigment epithelium (RPE). The thickness of the PRL was measured by measuring the distance between the inner edge of the external limiting membrane (ELM), a medium reflective layer, and the inner edge of the RPE. The ONL thickness was defined as the distance between the ILM and the ELM. The vertical thickness of the OSs of the photoreceptors was measured as the distance between the high reflective layer, which corresponded to the IS/OS junction and the inner edge of RPE. The vertical thickness of the ISs of the photoreceptors was the distance between the medium reflective layer (ELM) and the IS/OS junction. The OS horizontal diameter was measured as the width of the hyper reflective area, below the IS/OS junction, in the foveal area (Figure 1). All the measurements were calculated in microns.

For the total foveal thickness and thickness of the ONL, besides the foveal center, further measurements were obtained at 10 points, five on each side of the foveal center, along all the six radial scans (Figure 2). The thickness of the PRL decreases away from the foveal center, which makes the thickness measurements less accurate. Hence, for calculating the thickness of PRL, the vertical thickness of OS and the vertical thickness of ISs, only two more points were measured on each side of the foveal center, along all the six radial scans (Figure 3). OS horizontal diameter was measured along all the six radial scans. All these measurements were taken separately in right as well as left eyes of seven cases with and seven age-matched controls.

\section{FM 100 test}

The color vision was tested using the FM 100 hue test. No limit was placed on the time taken for the test. None of the patients had any previous experience with the test. The test was carried out monocularly. No near correction was required by any subject as all were $<30$ years of age. The caps were presented to the observers in four sets of 21 or 22 caps as described by Farnsworth. The order of 


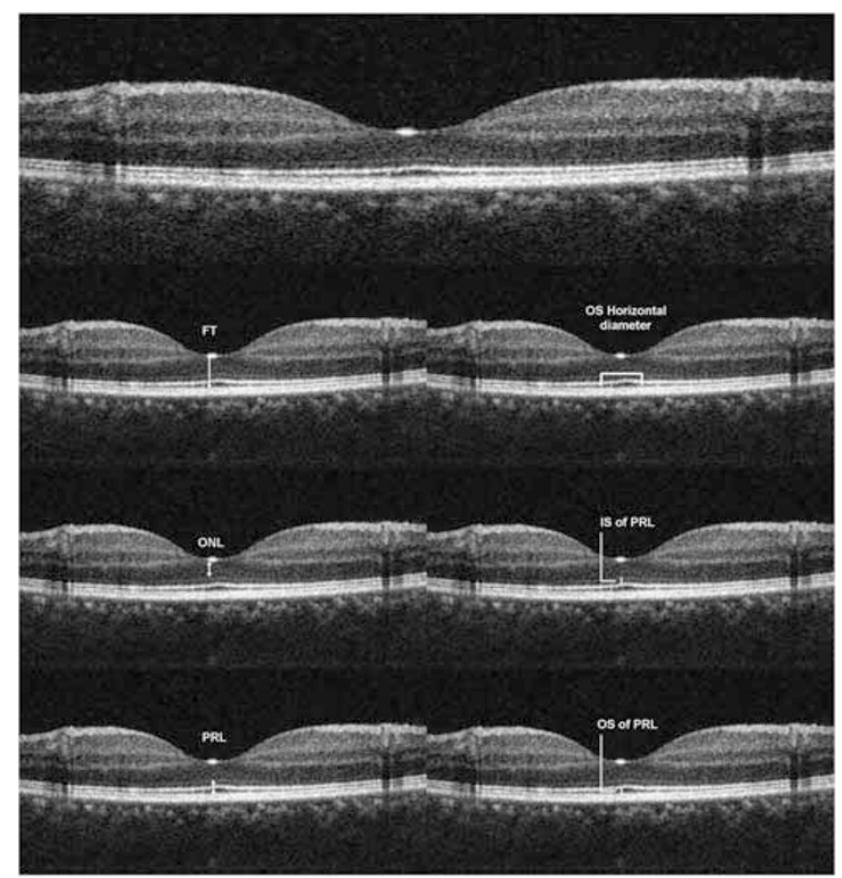

Figure 1 SD-OCT showing measurement parameters in study subjects. FT; foveal thickness; ONL, outer nuclear layer; PRL, photoreceptor layer; OS, outer segment; IS, inner segment.

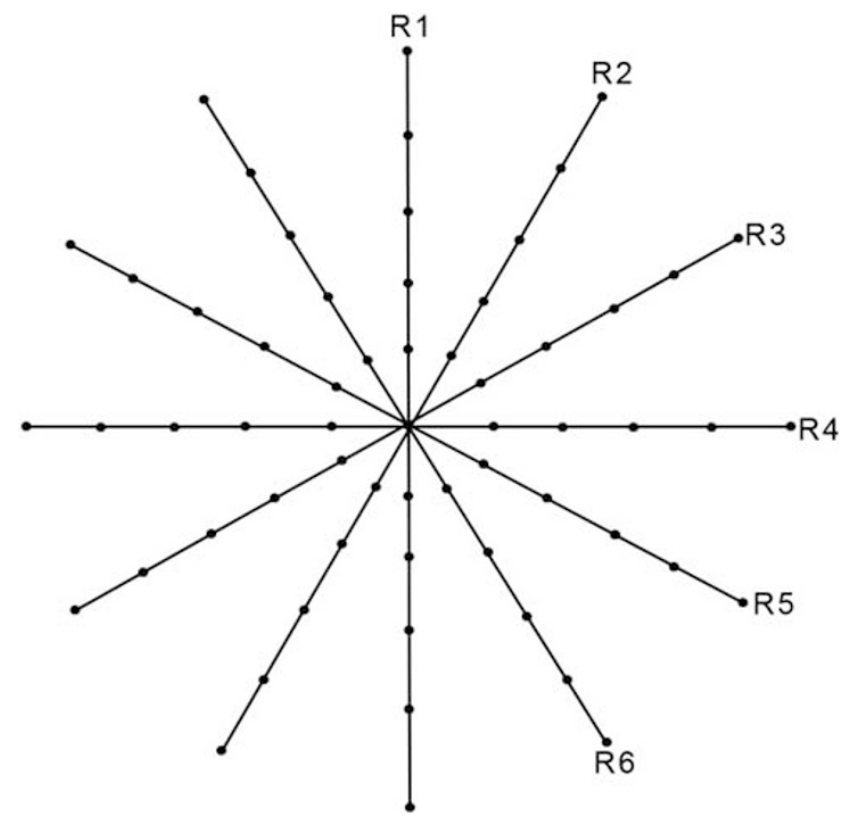

Figure 2 Diagram representing the eleven points measured along each of the six radial scans for foveal thickness and outer nuclear layer thickness.

presenting the boxes varied randomly. In all subjects, the scoring was calculated by an automatic electronic system. The error for each color cap was calculated and plotted according to the method of Farnsworth, ${ }^{6}$ and the total error score (TES), calculated as root mean square ${ }^{15}$, for all 85 caps was computed. The TES was judged as abnormal if it fell outside the 95th percentile for age, as published by Verriest et al, ${ }^{16}$ for monocular testing without previous binocular experience, and normal otherwise. The moment of inertia analysis yielded the TES and three factors which quantified the cap arrangements: the first was the confusion angle which 


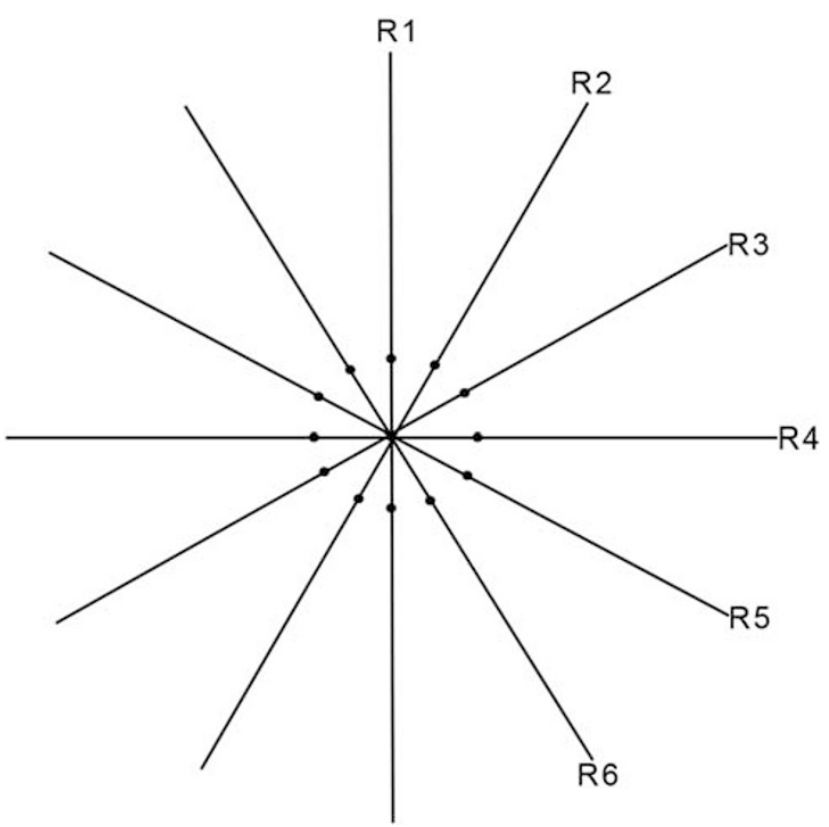

Figure 3 Diagram representing the three points measured along each of the six radial scans for photoreceptor layer, outer segment thickness and inner segment thickness.

identified the type of color defect, the second was the Confusion index which quantified the degree of color loss relative to a perfect arrangement of caps, and the third was the Selectivity index which quantified the amount of polarity or lack of randomness in a cap arrangement. The major and minor radii were also calculated. ${ }^{17}$

The 100-hue test score charts were also analyzed to determine any polarity of error score. In an attempt to remove subjectivity from the identification of any such cases, a mathematical method, based on the use of Fourier series, was developed and applied to the test data. ${ }^{18}$

The error scores were calculated as square root, according to Kinnear et al, ${ }^{15}$ the axis were recorded according to Smith $e t a l,{ }^{19}$ and the results were drawn according to Kinnear et al. ${ }^{15}$

The error scores changed with age. ${ }^{20}$ Hence, an age-matched control was studied for each CVD case to establish normal values for comparison.

\section{Microperimetry}

MP was performed in the mydriatic state using Goldmann size III stimuli, 4-2 threshold strategy, and a white background with an intensity of four apostilbs. An automated program was used using 33 stimulus points, which were projected in the central $20^{\circ}$ of fundus. The retinal image was captured, initially, using the infrared camera. Then, the location of the blind spot was confirmed. The blind spot was used to check the false-positive responses. The stimuli were projected one at a time (duration of stimuli being $200 \mathrm{~ms}$ ), and the patient was asked to respond to every stimulus seen by him by pressing a hand-held button. A well-defined reference mark in the retina was chosen by the examiner to help track the saccadic eye movements. The false-negative responses were tested once in every minute during the test, to test the reliability of the test results. The data was registered on a color picture and the retinal sensitivity was expressed in decibels.

\section{Exclusion criteria}

Subjects with acquired CVD, having vision less than 6/9 Snellen's equivalent and a refractive error greater than +6.00 or less than -6.0 diopters were excluded from the study.

\section{Statistical analysis}

Statistical analyses were performed using SPSS (Statistical Package for Social Sciences, version 14.0, Chicago, IL, USA). The results were expressed as mean + SD if the variables were continuous, and as percentage, if categorical. The level of statistical significance for $P$-value was $<0.05$. The data were examined and found not to be normally distributed. Therefore a Mann-Whitney $U$-test was used for comparison and the results are expressed using the 
median and interquartile range as the descriptive statistic.

\section{Results}

Table 1 shows the baseline data of the study patients, including their FM 100 test results. The mean age of the cases was 25 years (21-30) and the mean age of controls was 24.4 years (20-30). The median visual acuity for the fourteen eyes, from seven patients with CVD, used for correlation analysis was logMAR 0 or 20/20 Snellen equivalent. The mean TES for the cases was 138.29 by classical method and 6.33 by moment of inertia method. The confusion and selectivity index were 2.1 and 1.5 , respectively.

Table 2 shows the mean thickness of the different layers in cases and controls as seen on the SD-OCT and the mean retinal and foveal sensitivity as seen on the MP.

Table 1 Color vision data as on FM 100 hue test in cases with CVD and controls

\begin{tabular}{lcc}
\hline & Controls $(\mathrm{n}=14)$ & Cases $(\mathrm{n}=14)$ \\
\hline Age (years) & 24.4 & $25.00 \pm 2.83$ \\
Median BCVA (logMAR) & 0 & 0 \\
TES classical & $30.8 \pm 17.2$ & $138.29 \pm 61.53$ \\
TES moment of inertia & $3.8 \pm 0.4$ & $6.33 \pm 1.31$ \\
Mean Ishihara error & 0 & $13.57 \pm 2.65$ \\
Angle & $60.5 \pm 10.8$ & $14.4 \pm 23.6$ \\
Major radius & $3.1 \pm 0.4$ & $5.2 \pm 1.1$ \\
Minor radius & $2.2 \pm 0.2$ & $3.6 \pm 0.8$ \\
Confusion index & $1.2 \pm 0.1$ & $2.1 \pm 0.4$ \\
Selectivity Index & $1.4 \pm 0.2$ & $1.5 \pm 0.2$ \\
\hline
\end{tabular}

Abbreviations: BCVA, best-corrected visual acuity; CVD, color vision deficiency; $\log M A R, \log$ of the minimum angle of resolution; TES, total error score.
When compared between CVD cases and controls, the difference in the foveal thickness $(P=0.482$ for the right eye, $P=0.848$ for the left eye), the thickness of PRL ( $P=0.805$ for the right eye, $P=1.000$ for the left eye), the thickness of ONL $(P=0.535$ for the right eye, $P=0.383$ for the left eye), thickness of OS ( $P=0.710$ for the right eye, $P=0.535$ for the left eye), and the thickness of IS ( $P=0.898$ for the right eye, $P=0.443$ for the left eye) was not statistically significant. However, OS horizontal diameter was less in left eye of the cases with CVD when compared with controls ( $P=0.338$ for the right eye, $P=0.009$ for the left eye). Likewise, the mean retinal sensitivity $(P=0.902$ for the right eye, $P=1.000$ for the left eye) and foveal sensitivity ( $P=0.805$ for the right eye, $P=0.073$ for the left eye) was similar between CVD cases and controls.

Table 3 shows the detailed comparison of foveal thickness, PRL thickness and ONL thickness between each case and its age-matched control. In comparison with age-matched control, ONL thickness was more in the right eye of one case $(P<0.0001)$, and less in left eye of three cases $(P=0.045 ; P=0.002 ; P=0.020)$ and more in right eye of one case $(P=0.001)$. There was no significant difference between the measurements of foveal thickness and PRL thickness.

Table 4 shows the detailed comparison of OS thickness, IS thickness and OS horizontal diameter between each case and its age-matched control. In comparison with age-matched control, IS thickness was less in the right eye of one case $(P=0.046)$, and less in left eye of one case $(P=0.046)$. Likewise, when compared with age-matched control, OS horizontal diameter was lesser in the right eye of four cases $(P=0.010 ; P=0.004 ; P=0.004$, and $P=0.003)$ and left eye of five cases $(P=0.004 ; P=0.010$; $P=0.004 ; P=0.006$ and $P=0.006$ ). There was no significant difference between the measurements of OS thickness between CVD cases and controls.

Table 2 Thickness of retinal layers on SD-OCT and retinal sensitivity on MP in cases with CVD and controls

\begin{tabular}{|c|c|c|c|c|c|c|}
\hline & \multicolumn{3}{|c|}{ Right eye } & \multicolumn{3}{|c|}{ Left eye } \\
\hline & Cases & Controls & $\mathrm{P}$ & Cases & Controls & $\mathrm{P}$ \\
\hline & Median (IQR) & Median (IQR) & & Median (IQR) & Median (IQR) & \\
\hline Foveal thickness & $225.0(32.4)$ & $217.2(27.9)$ & 0.482 & $225.6(21.1)$ & $213.9(33.4)$ & 0.848 \\
\hline PRL thickness & $57.3(3.6)$ & $56.8(5.4)$ & 0.805 & $58.6(4.3)$ & $58.9(5.5)$ & 1.000 \\
\hline ONL thickness & $75.6(16.5)$ & 73.7 (17.6) & 0.535 & 74.6 (15.7) & 76.3 (16.3) & 0.383 \\
\hline OS thickness & 30.7 (3.6) & $29.3(1.8)$ & 0.710 & $29.9(2.6)$ & $29.0(4.0)$ & 0.535 \\
\hline IS thickness & $25.4(5.0)$ & $28.1(3.9)$ & 0.898 & $28.4(4.3)$ & $29.3(2.4)$ & 0.443 \\
\hline OS horizontal diameter & $288.7(134.0)$ & $329.2(137.2)$ & 0.338 & $258.5(157.7)$ & $394.7(114.0)$ & 0.009 \\
\hline Mean retinal sensitivity & $17.6(3.0)$ & $17.5(2.0)$ & 0.902 & $17.3(1.2)$ & $17.4(1.3)$ & 1.000 \\
\hline Foveal sensitivity & $20.0(4.0)$ & $20.0(6.0)$ & 0.805 & $18.0(6.0)$ & 20.0 (NA) & 0.073 \\
\hline
\end{tabular}

Abbreviations: IQR, interquartile range; IS, inner segment; MP, microperimetry; ONL, outer nuclear layer; OS, outer segment; PRL, photoreceptor layer; SD-OCT, spectral domain optical coherence tomography.

$P$-values in bold represent significance of $<0.05$. 
Table 3 Case and control wise comparison of foveal thickness, ONL thickness and PRL thickness

\begin{tabular}{|c|c|c|c|c|c|c|c|c|c|}
\hline & \multicolumn{3}{|c|}{ Foveal thickness } & \multicolumn{3}{|c|}{ ONL thickness } & \multicolumn{3}{|c|}{ PRL thickness } \\
\hline & Case & Control & $\mathrm{P}$ & Case & Control & $\mathrm{P}$ & Case & Control & $\mathrm{P}$ \\
\hline & Median (IQR) & Median (IQR) & & Median (IQR) & Median (IQR) & & Median (IQR) & Median (IQR) & \\
\hline \multicolumn{10}{|l|}{ Right eye } \\
\hline Case 1 vs control 1 & $225.2(52.5)$ & $223.0(78.3)$ & 0.718 & $80.0(26.0)$ & $80.0(21.9)$ & 0.694 & 58.7 (NA) & 57.3 (NA) & 0.827 \\
\hline Case 2 vs control 2 & $233.7(99.5)$ & $257.0(74.0)$ & 0.412 & $73.5(13.0)$ & $78.3(33.5)$ & 0.450 & 54.7 (NA) & 53.2 (NA) & 0.376 \\
\hline Case 3 vs control 3 & $237.7(89.2)$ & $215.7(81.5)$ & 0.139 & $100.7(5.5)$ & $73.8(17.6)$ & $<0.0001$ & 59.7 (NA) & 58.8 (NA) & 0.248 \\
\hline Case 4 vs control 4 & $188.2(69.5)$ & $216.2(82.1)$ & 0.250 & $71.7(20.3)$ & $80.0(18.8)$ & 0.309 & 54.5 (NA) & 61.8 (NA) & 0.275 \\
\hline Case 5 vs control 5 & $210.2(81.7)$ & $198.5(56.0)$ & 0.670 & $61.3(24.2)$ & $59.7(23.5)$ & 0.818 & 57.5 (NA) & 55.2 (NA) & 0.513 \\
\hline Case 6 vs control 6 & $237.8(86.1)$ & $227.2(81.8)$ & 0.490 & $83.8(10.7)$ & $66.7(33.5)$ & 0.061 & 54.8 (NA) & 54.8 (NA) & 0.822 \\
\hline Case 7 vs control 7 & $200.0(60.9)$ & $162.3(80.0)$ & 0.108 & $62.8(31.3)$ & $64.2(12.3)$ & 0.818 & 50.8 (NA) & 51.8 (NA) & 0.376 \\
\hline \multicolumn{10}{|l|}{ Left eye } \\
\hline Case 1 vs control 1 & $227.5(71.8)$ & $228.7(83.9)$ & 0.768 & $77.2(23.7)$ & $81.5(29.5)$ & 0.375 & 60.8 (NA) & 59.6 (NA) & 0.376 \\
\hline Case 2 vs control 2 & $235.8(96.5)$ & $271.3(53.5)$ & 0.076 & $71.8(13.0)$ & $82.8(28.8)$ & 0.045 & 57.8 (NA) & 54.1 (NA) & 0.050 \\
\hline Case 3 vs control 3 & $239.5(88.3)$ & $215.5(74.2)$ & 0.250 & $96.7(7.7)$ & $70.8(23.7)$ & 0.001 & 58.5 (NA) & 59.4 (NA) & 0.827 \\
\hline Case 4 vs control 4 & $190.3(60.4)$ & $214.3(79.9)$ & 0.412 & $57.8(12.5)$ & $76.8(16.8)$ & 0.002 & 57.3 (NA) & 64.0 (NA) & 0.077 \\
\hline Case 5 vs control 5 & $216.3(81.7)$ & $203.2(49.1)$ & 0.818 & $61.7(18.2)$ & $61.0(29.7)$ & 0.870 & 58.7 (NA) & 58.9 (NA) & 0.827 \\
\hline Case 6 vs control 6 & $229.3(90.5)$ & $238.7(66.2)$ & 0.622 & $77.5(5.3)$ & $72.0(28.2)$ & 0.293 & 54.5 (NA) & 56.8 (NA) & 0.513 \\
\hline Case 7 vs control 7 & $206.7(59.7)$ & $182.2(75.6)$ & 0.178 & $53.3(20.9)$ & $69.8(8.0)$ & 0.020 & 49.2 (NA) & 50.2 (NA) & 0.268 \\
\hline
\end{tabular}

Abbreviations: IQR, interquartile range; NA, interquartile range not available because of less than four parameters; ONL, outer nuclear layer; PRL, photoreceptor layer.

$P$-values in bold represent significance of $<0.05$.

Table 4 Case and control wise comparison of OS thickness, IS thickness and OS horizontal diameter

\begin{tabular}{|c|c|c|c|c|c|c|c|c|c|}
\hline & \multicolumn{3}{|c|}{ OS thickness } & \multicolumn{3}{|c|}{ IS thickness } & \multicolumn{3}{|c|}{ OS horizontal diameter } \\
\hline & Case & Control & $\mathrm{P}$ & Case & Control & $\mathrm{P}$ & Case & Control & $\mathrm{P}$ \\
\hline & Median (IQR) & Median (IQR) & & Median (IQR) & Median (IQR) & & Median (IQR) & Median (IQR) & \\
\hline \multicolumn{10}{|l|}{ Right eye } \\
\hline Case 1 vs control 1 & 27.2 (NA) & 27.5 (NA) & 0.376 & 31.5 (NA) & 29.7 (NA) & 0.050 & 367.5 (NA) & 470.8 (NA) & 0.010 \\
\hline Case 2 vs control 2 & 29.5 (NA) & 26.8 (NA) & 0.275 & 26.0 (NA) & 26.3 (NA) & 0.513 & 342.5 (NA) & 273.7 (NA) & 0.025 \\
\hline Case 3 vs control 3 & 29.8 (NA) & 27.7 (NA) & 0.275 & 30.3 (NA) & 30.0 (NA) & 0.827 & 444.8 (NA) & 329.2 (NA) & 0.004 \\
\hline Case 4 vs control 4 & 29.0 (NA) & 32.5 (NA) & 0.275 & 25.5 (NA) & 29.2 (NA) & 0.046 & 254.8 (NA) & 416.0 (NA) & 0.004 \\
\hline Case 5 vs control 5 & 27.8 (NA) & 28.0 (NA) & 0.875 & 28.5 (NA) & 27.2 (NA) & 0.275 & 288.7 (NA) & 278.8 (NA) & 0.519 \\
\hline Case 6 vs control 6 & 30.3 (NA) & 25.3 (NA) & 0.275 & 24.5 (NA) & 27.7 (NA) & 0.121 & 233.5 (NA) & 370.7 (NA) & 0.004 \\
\hline Case 7 vs control 7 & 28.0 (NA) & 26.8 (NA) & 1.000 & 23.7 (NA) & 25.0 (NA) & 0.513 & 193.3 (NA) & 282.3 (NA) & 0.003 \\
\hline \multicolumn{10}{|l|}{ Left eye } \\
\hline Case 1 vs control 1 & 28.2 (NA) & 30.0 (NA) & 0.275 & 30.7 (NA) & 27.2 (NA) & 0.050 & 258.5 (NA) & 560.3 (NA) & 0.004 \\
\hline Case 2 vs control 2 & 31.5 (NA) & 24.7 (NA) & 0.050 & 26.3 (NA) & 28.3 (NA) & 0.050 & 375.8 (NA) & 319.7 (NA) & 0.078 \\
\hline Case 3 vs control 3 & 30.0 (NA) & 27.2 (NA) & 0.275 & 27.8 (NA) & 30.8 (NA) & 0.184 & 368.5 (NA) & 385.8 (NA) & 0.873 \\
\hline Case 4 vs control 4 & 29.2 (NA) & 31.0 (NA) & 0.275 & 28.2 (NA) & 32.3 (NA) & 0.127 & 317.3 (NA) & 437.7 (NA) & 0.010 \\
\hline Case 5 vs control 5 & 26.5 (NA) & 27.8 (NA) & 0.369 & 29.8 (NA) & 29.7 (NA) & 0.827 & 247.7 (NA) & 400.0 (NA) & 0.004 \\
\hline Case 6 vs control 6 & 29.7 (NA) & 26.0 (NA) & 0.275 & 24.8 (NA) & 29.5 (NA) & 0.046 & 210.8 (NA) & 323.7 (NA) & 0.006 \\
\hline Case 7 vs control 7 & 23.7 (NA) & 25.0 (NA) & 0.275 & 25.7 (NA) & 25.5 (NA) & 0.376 & 175.0 (NA) & 394.7 (NA) & 0.006 \\
\hline
\end{tabular}

Abbreviations: IQR, interquartile range; IS, inner segment; NA, interquartile range not available because of less than four parameters; OS, outer segments. $P$-values in bold represent significance of $<0.05$.

\section{Discussion}

CVD is a common disorder and has undoubted practical consequences in everyday life. ${ }^{21}$ In dichromats, the missing class of cones may be replaced by rods. In anomalous trichromats, the cones are known to be dysfunctional but there is no documentation of structural changes in retina in subjects with CVD. Barthelmes et $a l^{11}$ quantified the OCT images of the central retina in patients with two cone diseases- $\mathrm{BCM}$ and $\mathrm{ACH}-$ and compared it with healthy control individuals. The study reported a highly altered foveolar structure in both BCM 
and ACH. They observed that the foveal thickness, in patients with BCM, reduced significantly but there was no change in the thickness of the central retina in patients with ACH observed by OCT. Varsányi et al ${ }^{12}$ reported statistically significant reductions in total macular volume and in the thickness of the central retina in patients with $\mathrm{ACH}$. Carroll et al ${ }^{13}$ observed a partial thinning of the ONL in the retina of subjects having red-green CVD with C203R mutation compared with a normal ONL in the subjects with LIAVA mutation. Lorenz et $a l^{22}$ reported the loss of retinal thickness because of the loss of stratification in the inner and OS layers and thinning of the ONL in patients having retinal dystrophy with some RPE65 missense mutations.

In the foveal retina, where only cones are concentrated, the cell bodies of cones are layered in oblique columns below the ELM. The OS are filled with stacks of membranes containing the visual pigment molecules and the IS contain mitochondria, ribosomes, and endoplasmic reticulum. In normal photoreceptors, opsin is synthesized in the rough endoplasmic reticulum, packaged into vesicles in the Golgi apparatus, transported in membrane vesicles, through the IS cytoplasm to the connecting cilium, and inserted into the newly formed membranes at the base of the OS. ${ }^{23}$ The photoreceptors synthesize large amounts of opsin and other OS proteins, a process balanced in part by periodic shedding of the OS tips which are phagocytosed and degraded by the RPE. ${ }^{24}$

Experimental studies in animals have demonstrated a significant decrease in the rate of rod OS disk synthesis in photoreceptor cells damaged by UVA light which is reported to be seen as shortening of OS. ${ }^{25}$ Rod OS shortening is also a consistent histopathological finding in the early stages of all genetic forms of RP including autosomal dominant RP caused by rhodopsin mutations. ${ }^{26,27}$ This morphological change correlates with the RP patient's decreased rod-mediated function and indicates that renewal of OS is probably diminished. ${ }^{28} \mathrm{In}$ the case of rhodopsin mutations, it has been suggested that the abnormal opsin may fail to reach the OS because it is not recognized for transport out of the endoplasmic reticulum or Golgi apparatus or is recognized as a foreign molecule and degraded by lysosomal activity in the IS. ${ }^{29-31}$ Mutations affecting an asparagine residue at position 1 or 04 in the N-terminus of rhodopsin may interfere with glycosylation and incorporation of the molecule into forming OS disks. ${ }^{32}$

We hypothesized that if similar findings are extrapolated to the cones in CVD, some of the pigment mutations in cones may interfere similarly with the incorporation of mutated cone color pigment into the forming disks, thus resulting into alterations in the vertical or horizontal thickness of OS. We measured the total foveal thickness, thickness of the ONL, thickness of the PRL, vertical thickness of OS and IS, and OS horizontal diameter in subjects with red-green CVD as identified on the FM 100 test.

However, on analyzing the results of multiple measurements taken across the foveal pit in many locations, we did not find any significant change in the thickness of OS and other retinal layers in cases with CVD. The morphology of foveal pit, though, was found to be different among cases and controls. The diameter of the foveal pit was found to be narrower (narrower OS horizontal diameter) in subjects with CVD in comparison with the normal, though, this was not the original hypothesis of the present study.

Yuodelis and Hendrickson ${ }^{33}$ reported quantitative measures of foveal development taken from pre- and postnatal human retinas. They described that before birth the rod-free zone or foveola was over $1000 \mu \mathrm{m}$ in diameter, but it became progressively narrower after birth because of a central ward migration of cones, reaching the adult diameter of $650-700 \mu \mathrm{m}$ by 45 months of age. Postnatally, foveolar cone development was characterized by maturation, elongation, and an increase in packing density. During this time the foveolar cone developed both its OS and basal axon process (fiber of Henle), resulting in an increase of foveolar cone density. Similar findings were also reported by Hendrickson. ${ }^{34}$

It might be possible that the subjects with congenital red-green CVD, owing to presence of mutations in the cone pigment or dysfunctional cones, may not be undergoing the mentioned structural changes in the foveal configuration. Blue cones are absent in the fovea. ${ }^{35}$ Hence, the smaller foveal pit as observed in the subjects with congenital CVD in this study, can be a result of smaller number of red-green cones, or smaller individual diameter of cones. However, this hypothesis needs confirmation by further investigations.

The main shortcoming of our study is the small sample size. Another shortcoming is that we did not do a genetic analysis, which could have shown the type of mutation in our subjects with CVD having normal retinal thickness measurements.

In conclusion, in subjects with red-green CVD, we observed normal thickness of different retinal layers in macula and narrower OS horizontal diameter in the left eye of CVD subjects. The observation of a narrower foveal pit in subjects with congenital CVD is interesting and is a good stimulus for future studies. Studies involving larger database and comparing the structural changes in the retina in dichromats and anomalous trichromats are needed. The correlation of OCT findings with genetic analysis in CVD subjects will provide further insight. 


\section{Summary}

\section{What was known before}

- Red-green color vision deficiency (CVD) is characterized by the absence of either the long or the medium cone function. The structural changes in the central retina on optical coherence tomography (OCT) in patients with two cone diseases-blue-cone monochromatism, and achromatopsia have been reported as a highly altered foveolar structure in both diseases. However, little is known about the structural changes in the retina in subjects with congenital CVD, which is also characterized by the lack of functioning cones.

\section{What this study adds}

- Spectral domain OCT demonstrated normal total foveal thickness, normal thickness of outer nuclear layer, normal thickness of the photoreceptor layer, normal vertical thickness of the outer segments (OS), normal vertical thickness of inner segments, and reduced OS horizontal diameter in cases with CVD when compared with controls. The mean retinal and foveal sensitivity was similar between cases and controls. The observation of a narrower foveal pit in subjects with congenital CVD is interesting and is a good stimulus for future studies.

\section{Conflict of interest}

The authors declare no conflict of interest.

\section{References}

1 Peacock G. Life of Thomas Young: M. D., F.R.S., Ec., and One of the Eight Foreign Associates of the National Institute of France. John Murray: London, 1855.

2 Scheibner H, Kellermann FJ, Boll M. [Protanopia and protanomaly]. Ber Zusammenkunft Dtsch Ophthalmol Ges 1972; 71: 522-531.

3 Scheibner H, Boll M. [Deuteranopia and deuteranomaly]. Albrecht Von Graefes Arch Klin Exp Ophthalmol 1972; 185(2): 145-160.

4 Nathans J, Thomas D, Hogness DS. Molecular genetics of human color vision: the genes encoding blue, green, and red pigments. Science 1986; 232: 193-202.

5 Birch J. Diagnosis of Defective Colour Vision, 2nd edn. Oxford University Press: Edinburgh, 1998.

6 Farnsworth D. The Farnsworth-Munsell 100-hue and dichotomous tests for colour vision. J Opt Soc Am 1943; 33 568-578.

7 Wojtkowski M, Bajraszewski T, Targowski P, Kowalczyk A. Real-time in vivo imaging by high-speed spectral optical coherence tomography. Opt Lett 2003; 28: 1745-1747.

8 Wojtkowski M, Bajraszewski T, Gorczyńska I, Targowski P, Kowalczyk A, Wasilewski W et al. Ophthalmic imaging by spectral optical coherence tomography. Am J Ophthalmol 2004; 138: 412-419.

9 Chen TC, Cense B, Pierce MC, Nassif N, Park BH, Yun SH et al. Spectral domain optical coherence tomography: ultrahigh speed, ultra-high resolution ophthalmic imaging. Arch Ophthalmol 2005; 23: 1715-1720.

10 Kiernan DF, Mieler WF, Hariprasad SM. Spectral-domain optical coherence tomography: a comparison of modern high-resolution retinal imaging systems. Am J Ophthalmol 2010; 149: 18-31.

11 Barthelmes D, Sutter FK, Kurz-Levin MM, Bosch MM, Helbig H, Niemeyer $G$ et al. Quantitative analysis of OCT characteristics in patients with achromatopsia and bluecone monochromatism. Invest Ophthalmol Vis Sci 2006; 47: 1161-1166.

12 Varsányi B, Somfai GM, Lesch B, Vámos R, Farkas A. Optical coherence tomography of the macula in congenital achromatopsia. Invest Ophthalmol Vis Sci 2007; 48: 2249-2253.

13 Carroll J, Baraas RC, Wagner-Schuman M, Rha J, Siebe CA, Sloan $\mathrm{C}$ et al. Cone photoreceptor mosaic disruption associated with Cys203Arg mutation in the M-cone opsin Proc Natl Acad Sci USA 2009; 106(49): 20948-20953.

14 Ishikawa H, Gürses-Ozden R, Hoh ST, Dou HL, Liebmann JM, Ritch R. Grayscale and proportion-corrected optical coherence tomography images. Ophthalmic Surg Lasers 2000; 31: 223-228.

15 Kinnear PR. Proposals for scoring and assessing the 100-hue test. Vision Res 1970; 10: 423-433.

16 Verriest G, van Laethem J, Uvijls A. A new assessment of the normal ranges of the Farnsworth-Munsell 100-hue test scores. Am J Ophthalmol 1982; 93: 635-642.

17 Vingrys AJ, King-Smith PE. A quantitative scoring technique for panel tests of color vision. Invest Ophthalmol Vis Sci 1988; 29: 50-63.

18 Allan D. Fourier analysis and the Farnsworth-Munsell 100-hue test. Ophthalmic Physiol Opt 1985; 5: 337-342.

19 Smith VC, Pokorny J, Pass AS. Color-axis determination on the Farnsworth-Munsell 100-hue test. Am J Ophthalmol 1985; 100: 176-182.

20 Pinckers A. Color vision and age. Ophthalmologica 1980; 181: 23-30.

21 Steward SM, Cole BL. What do colour vision defectives say about everyday tasks? Optom Vis Sci 1989; 66: 288-295.

22 Lorenz B, Poliakov E, Schambeck M, Friedburg C, Preising MN, Redmond TM. A comprehensive clinical and biochemical functional study of a novel RPE65 hypomorphic mutation. Invest Ophthalmol Vis Sci 2008; 49: 5235-5242.

23 Deretic D, Papermaster DS. The role of small G-proteins in the transport of newly synthesized rhodopsin. Prog Retin Eye Res 1994; 14: 249-265.

24 Bok D. Retinal photoreceptor-pigment epithelium interactions. Friedenwald lecture. Invest Ophthalmol Vis Sci 1985; 26: 1659-1694.

25 Rapp LM, Fisher PL, Dhindsa HS. Reduced rate of rod outer segment disk synthesis in photoreceptor cells recovering from UVA light damage. Invest Ophthalmol Vis Sci 1994; 35: 3540-3548.

26 Li ZY, Jacobson SG, Milam AH. Autosomal dominant retinitis pigmentosa caused by the threonine-17-methionine rhodopsin mutation: retinal histopathology and immunocytochemistry. Exp Eye Res 1994; 58: 397-408.

27 Milam AH, Li ZY, Cideciyan AV, Jacobson SG. Clinicopathologic effects of the Q64ter rhodopsin mutation in retinitis pigmentosa. Invest Ophthalmol Vis Sci 1996; 37: 753-765.

28 Milam AH, Li ZY, Fariss RN. Histopathology of the human retina in retinitis pigmentosa. Prog Retin Eye Res 1998; 17: 175-205.

29 Bird AC. Investigation of disease mechanisms in retinitis pigmentosa. Ophthalmic Paediatr Genet 1992; 13: 57-66. 
30 Dryja TP. Doyne Lecture. Rhodopsin and autosomal dominant retinitis pigmentosa. Eye (Lond) 1992; 6(Pt 1): 1-10.

31 Manoil C, Traxler B. Membrane protein assembly: genetic, evolutionary and medical perspectives. Annu Rev Genet 1995; 29: 131-150.

32 Berson EL. Ocular findings in a form of retinitis pigmentosa with a rhodopsin gene defect. Trans Am Ophthalmol Soc 1990; 88: 355-388.
33 Yuodelis C, Hendrickson A. A qualitative and quantitative analysis of the human fovea during development. Vision Res 1986; 26: 847-855.

34 Hendrickson AE. Primate foveal development: a microcosm of current questions in neurobiology. Invest Ophthalmol Vis Sci 1994; 35: 3129-3133.

35 Rowe MH. Trichromatic color vision in primates. News Physiol Sci 2002; 17: 93-98. 